Acta Crystallographica Section B

Structural

Science

ISSN 0108-7681

Richard E. Marsh ${ }^{\mathrm{a} *}$ and Robert A. Sparks ${ }^{\mathbf{b}}$

\author{
${ }^{a}$ The Beckman Institute, California Insti- \\ tute of Technology, Pasadena, CA \\ 91125, USA, and ${ }^{\mathbf{b}} 1800$ Pearl Street \\ West, Apartment 4, Tillamook, Oregon \\ 97141, USA
}

Correspondence e-mail: rem@xray.caltech.edu

\section{Space-group changes: a revision to a revision}

The space group for the entry under the reference

Received 25 May 2001 codes FEBMUU and FEBMUU01 in the Cambridge Accepted 24 July 2001 Structural Database (1992) should be further corrected to space group $C 2 / c$ rather than $P 1$ (FEBMUU) or $C 2$ (FEBMUU01).

\section{Comment}

In a recent paper discussing incorrect spacegroup assignments, Marsh (1995) reported that the space group of the compound disodium $\left(\mu_{2}\right.$-oxo $)$ bis $\left(\mu_{2}\right.$-xanthopterine- $\left.N, O, O^{\prime}\right)$ bis(dioxomolybdenum) dimethylsulfoxide tetrahydrofuran solvate, which was listed as triclinic, space group $P 1$ in both the original paper (Burgmayer \& Stiefel, 1986) and in the Cambridge Structural Database (1992; CSD) under the Refcode FEBMUU, should be revised to monoclinic, $C 2$. However, he failed to realise that the value of $Z$, as given both in the CSD listing and in the original paper, was 2 , but that coordinates were reported for only a single molecule (which has symmetry $C_{2}$ ); neither did he notice that the density would be unreasonable for $Z=1$. Thus, he failed to appreciate that this was one of the many instances, in both the crystallographic literature and in the CSD (Marsh, 1999), where the space group $P \overline{1}$ has been incorrectly reported as $P 1$ - perhaps because of problems in creating an 'overline' symbol. (These mistakes have been corrected in recent releases of the CSD.)

When one of us (RAS) was checking the program FINDSYM (R. A. Sparks, unpublished), which searches lists of coordinates for additional symmetry elements, he noted that the original coordinates (FEBMUU), when applied to space group $P 1$, resulted in a structure with large voids, and that the voids disappeared and a reasonable packing arrangement resulted when he presumed the space group to be $P \overline{1}$. In addition, he noted that additional symmetry was present and that the structure should be described in the monoclinic space group $C 2 / c$, rather than the $C 2$ reported by Marsh (1995) and in FEBMUU01. Atom coordinates for the $C 2 / c$ description can be obtained from the $C 2$ coordinates by incrementing all $z$ values by 0.25 .

We further note that, again in Table 1 of Marsh (1995), the 'original' space groups of FEBMUU, HZPYCU and LACFAW were misprinted as $P \overline{1}$ rather than $P 1$.

Note added in proof. On 29 July 2001 Robert Sparks and his wife Nonie died as the result of an automobile accident near their house in Tillamook, Oregon.

After receiving the proof I was informed by Dr Larry Henling that the space group of this compound, FEBMUU, has already been revised from $P 1$ to $C 2 / c$ by Professor Frank Herbstein [(1997), Acta Cryst. B53, 968-975]; the revision has not yet been included in the CSD. Rather than withdrawing this note, I have requested that it be published as a tribute to Bob Sparks and to the large amount of time and insight that he contributed, during the past two years, to the development of FINDSYM.

\section{References}

Burgmayer, S. J. N. \& Stiefel, E. I. (1986). J. Am. Chem. Soc. 108, 8310-8311.

Cambridge Structural Database (1992). CSD User's Manual. Cambridge Crystallographic Data Centre, 12 Union Road, Cambridge, England.

Marsh, R. E. (1995). Acta Cryst. B51, 897-907.

Marsh, R. E. (1999). Acta Cryst. B55, 931-936. 